\title{
Weiner Polynomials for Generalization of Distance for Some Special Graphs
}

Ali A. Ali

aliazizali1933@yahoo.com
Ahmed M. Ali

ahmedgraph@uomosul.edu.iq

College of Computer Sciences and Mathematics

University of Mosul, Iraq

Received on: 08/03/2006

\section{ABSTRACT}

Accepted on: 04/04/2006

The minimum distance of a vertex $v$ to an $(n-1)-$ set of vertices of a graph $\mathrm{G}$ is defined as :

$\mathrm{d}_{\mathrm{n}}(\mathrm{v}, \mathrm{S})=\min \{\mathrm{d}(\mathrm{v}, \mathrm{u}): \mathrm{u} \in \mathrm{S}\}$.

The $n$-Wiener polynomial for this distance of a graph $\mathrm{G}$ is defined as

$$
\mathrm{W}_{\mathrm{n}}(\mathrm{G} ; \mathrm{x})=\sum_{\mathrm{k}=0}^{\delta_{\mathrm{n}}} \mathrm{C}_{\mathrm{n}}(\mathrm{G}, \mathrm{k}) \mathrm{x}^{\mathrm{k}},
$$

where $C_{n}(G, k)$ is the number of order pairs $(v, S)$, such that $|S|=n-1$

$\mathrm{d}_{\mathrm{n}}(\mathrm{v}, \mathrm{S})=\mathrm{k}$,

and $\delta_{n}$ is the diameter for this minimum n-distance.

In this paper, the n-Wiener polynomials for some types of graphs such as complete graphs, bipartite graphs, star graphs, wheel graphs, path and cycle graphs are obtained.The n-Wiener index for each of these special graphs is given. Moreover, some properties of the coefficients of are established. $\mathrm{W}_{\mathrm{n}}(\mathrm{G} ; \mathrm{x})$

Keywords: n-distance, Wiener polynomial, Special graphs.

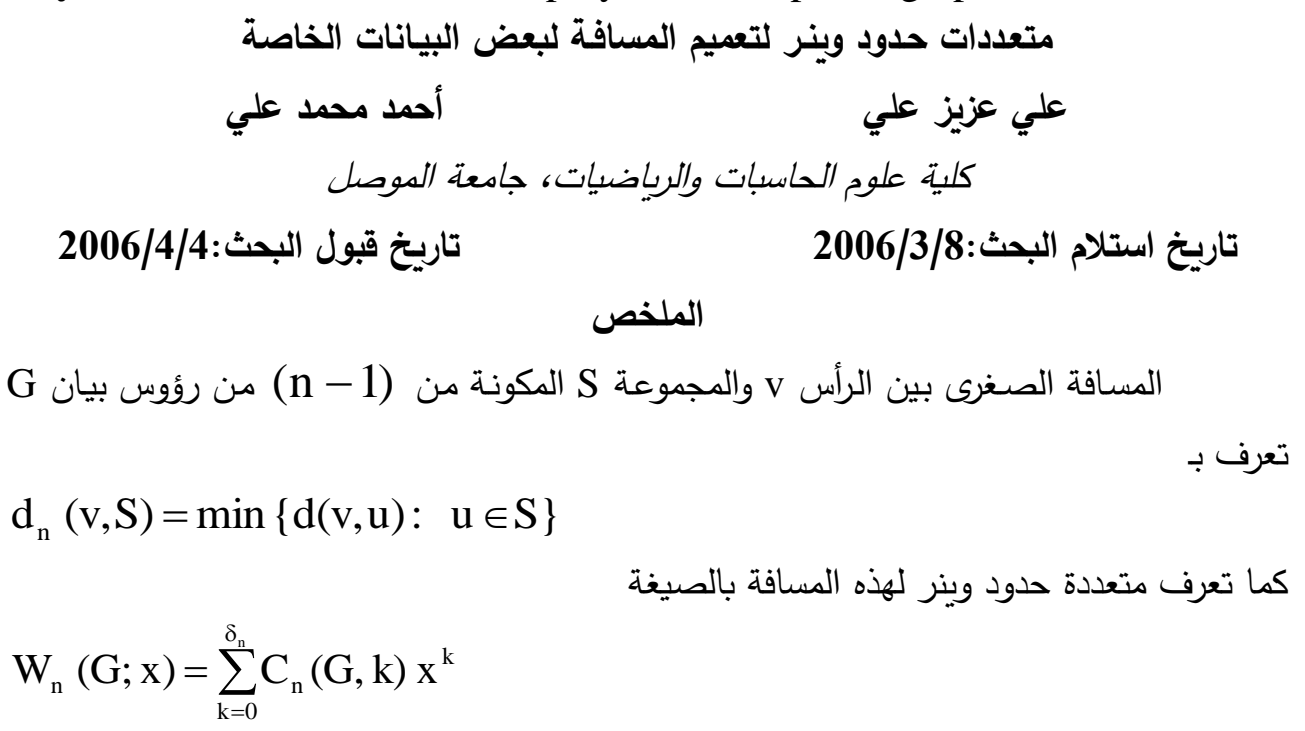




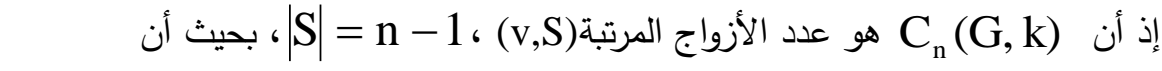
$\mathrm{d}_{\mathrm{n}}(\mathrm{v}, \mathrm{S})=\mathrm{k}$

علما بأن $\delta_{n}$ هو القطر بالنسبة إلى المسافة الصغرى. تضمن هذا البحث إيجاد متعددة حدود وينر -n لبعض أصناف البيانات الخاصة كالبيانات

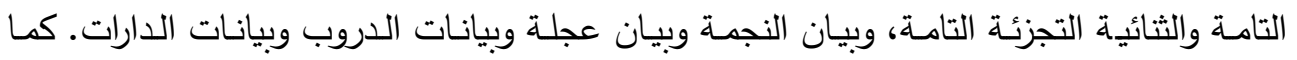

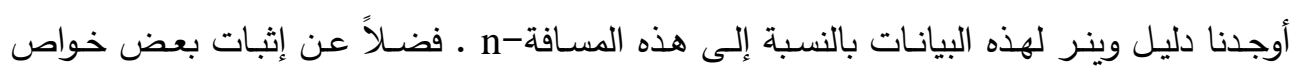
. W الكلمات المفتاحية : المسافة - n ، متعددة حدود وينر ، بيانات خاصة.

\section{Introduction}

لقد قام العديد من الباحثين بتعريف المسافة بين مجموعتين غير خاليتين من رؤوس بيان

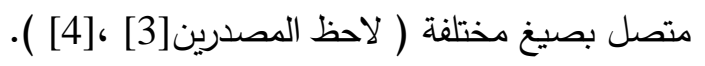

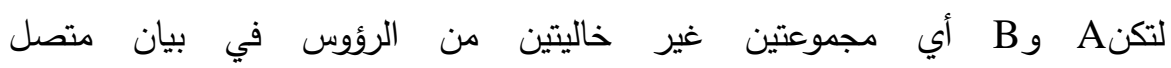
منتهى G = (V,E)، إذ أنه ليس من الضروري أن تكون المجموعتان A و B مختلفتين، فقد عرف بعض الباحثين المسافة بين مجموعتين A و B، في البيان Gبأساليب مختلفة نذكر بعضاًً منها. المسافة الصغرى (the minimum distance): من المجموعة A إلى المجموعة B في في $d_{\text {min }}(A, B)=\min \{d(a, b): a \in A, b \in B\}$

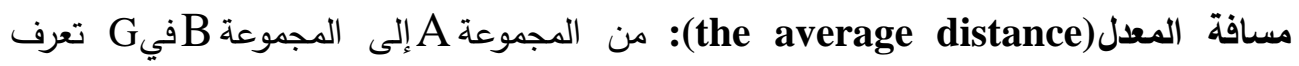
$\mathrm{d}_{\mathrm{ave}}(\mathrm{A}, \mathrm{B})=\frac{1}{|\mathrm{~A} \| \mathrm{B}|} \sum_{\mathrm{a} \in \mathrm{A}, \mathrm{b} \in \mathrm{B}} \mathrm{d}(\mathrm{a}, \mathrm{b})$

المسافة العظمى (the maximum distance): من المجموعة A إلى الهجوعة B في G

$$
\mathrm{d}_{\text {max }}(\mathrm{A}, \mathrm{B})=\max \{\mathrm{d}(\mathrm{a}, \mathrm{b}): \mathrm{a} \in \mathrm{A}, \mathrm{b} \in \mathrm{B}\}
$$

نلاحظ عندما تكون المجموعتان A A B ، أحاديتين فأن كلا من الأنواع الثلاثة من هذه

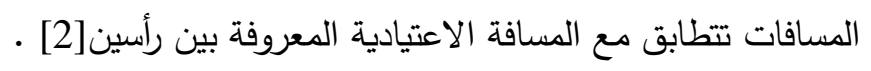


لأجل أن نعرف القطر ونصف القطر للبيان المتصل Gبالنسبة إلى كل من الأصناف الثلاثة للمسافة، نعرف أولا: الاختلاف المركزي-n لرأس (the n- eccentricity of a vertex v) (على أنه أكبر مسافة

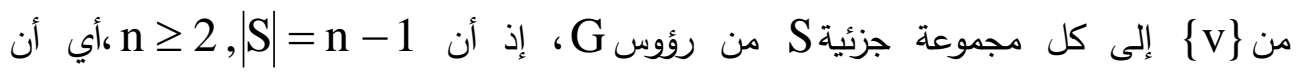

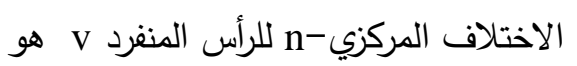
$\mathrm{e}_{\mathrm{M}}(\mathrm{v}, \mathrm{n})=\max \left\{\mathrm{d}_{\mathrm{M}}(\mathrm{v}, \mathrm{S}): \mathrm{S} \subseteq \mathrm{V},|\mathrm{S}|=\mathrm{n}-1\right\}$ إذ أن d هي دالة المسافة لأي من الأنواع الثلاثة ( إما .max أو .ave أو.min ) . نلاحظ أنه عندما تكون n=2 ، فأن التعاريف السابقة للمسافة تعطي تعريف الاختلاف

المركزي الاعتيادي للرأس vل [2].

$$
\text { ويمكن أن نعرف }
$$

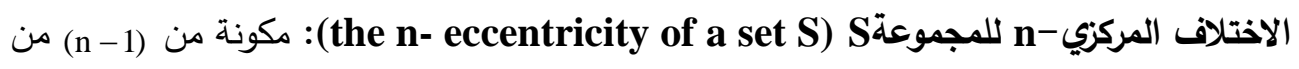

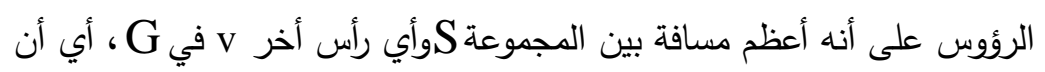
$\mathrm{e}_{\mathrm{M}}(\mathrm{S})=\max \left\{\mathrm{d}_{\mathrm{M}}(\mathrm{v}, \mathrm{S}): \mathrm{v} \in \mathrm{V}\right\}$

ونلاحظ أيضا أنه عندما تكون

$$
\text { الاختلاف المركزي الاعتيادي. }
$$

وأخيرا إن الأنواع الثثلثة من الاختلافات المركزية-n لرأس Vرتبطة مع الاختلاف المركزي

الاعتيادي بالعلاقة الآتية [4] •

$$
\mathrm{e}(\mathrm{v})-\mathrm{n}+1 \leq \mathrm{e}_{\text {min }}(\mathrm{v}, \mathrm{n}) \leq \mathrm{e}_{\mathrm{ave}}(\mathrm{v}, \mathrm{n}) \leq \mathrm{e}_{\max }(\mathrm{v}, \mathrm{n})=\mathrm{e}(\mathrm{v})
$$

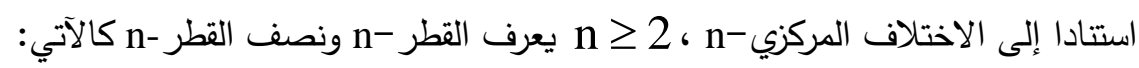

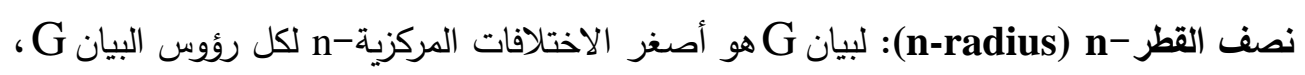

$$
\mathrm{n}-\operatorname{rad}_{\mathrm{M}}(\mathrm{G})=\min \left\{\mathrm{e}_{\mathrm{M}}(\mathrm{v}, \mathrm{n}): \forall \mathrm{v} \in \mathrm{V}\right\}
$$

وأن قطر-n-diameter) no هو أعظم الاختلافات المركزية-n لكل رؤوس

$$
\mathrm{n}-\operatorname{diam}_{\mathrm{M}}(\mathrm{G})=\max \left\{\mathrm{e}_{\mathrm{M}}(\mathrm{v}, \mathrm{n}): \forall \mathrm{v} \in \mathrm{V}\right\}
$$

ويمكن أن نعرف قطر -n ونصف القطر -n للبيان بالاعتماد على الاختلاف المركزي

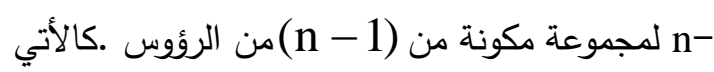




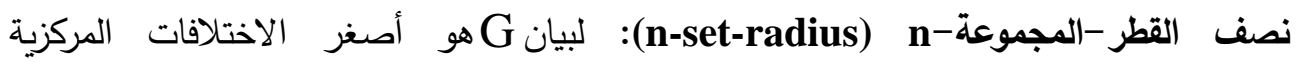

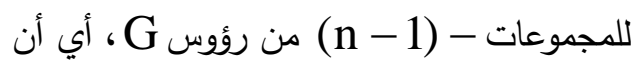

$\mathrm{n}-\mathrm{set}-\operatorname{rad}_{\mathrm{M}}(\mathrm{G})=\min \left\{\mathrm{e}_{\mathrm{M}}(\mathrm{S}), \mathrm{S} \subseteq \mathrm{V}\right\}$

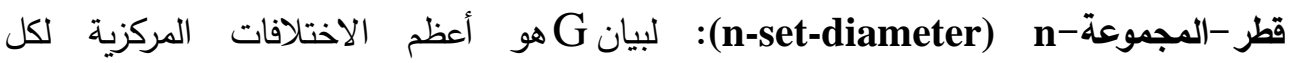

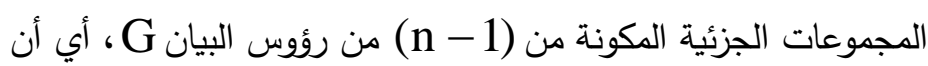
$\mathrm{n}-\mathrm{set}-\operatorname{diam}_{\mathrm{M}}(\mathrm{G})=\max \left\{\mathrm{e}_{\mathrm{M}}(\mathrm{S}), \mathrm{S} \subseteq \mathrm{V}\right\}$ واضح أن قطر -n وقطر -المجموعة-n يكونان متساويين دائما .

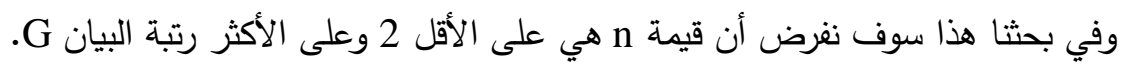

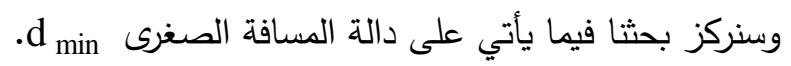

$$
\begin{aligned}
& \text { واضح أنه إذا كان }
\end{aligned}
$$

في الحقيقة أن d din تحقق الخواص الآتية على الدموعات الجزئية غير الخالية من

$$
\begin{aligned}
& d_{\text {min }}(A, B) \geq 0 \\
& d_{\text {min }}(A, B)=0 \quad \text { iff } \quad A \cap B \neq \phi \\
& d_{\text {min }}(A, B)=d_{\text {min }}(B, A)
\end{aligned}
$$

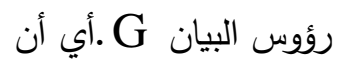$$
\mathrm{d}_{\text {min }}(\mathrm{A}, \mathrm{B})+\mathrm{d}_{\text {min }}(\mathrm{B}, \mathrm{C}) \geq \mathrm{d}_{\text {min }}(\mathrm{A}, \mathrm{C})
$$

ولكن المتباينة المثلثية (triangle inequality)

لا تتحقق بصورة عامة ، إذ أن B, A و C مجموعات جزئية من الرؤوس غير خالية في G [3] - ل

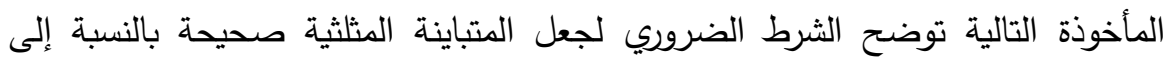

لتكن A و C مجموعتين جزئيتين غير خاليتين من مجموعة رؤوس G، وليكن رأسا

$$
d_{\text {min }}(A, b)+d_{\text {min }}(b, C) \geq d_{\text {min }}(A, C) \text {. }
$$

(2)بع خواص متعدات حدود وينر لتعميم المسافة

Some Properties of the Wiener Polynomials for Generalized Distance ستتقصر دراستنا في هذا البحث على دالة المسافة الصغرى فقط وعندما تكون المجموعةA أحادية.ولأجل ذلك نضع التعريف الأتي: 


\section{(2.1) : التعريف(5)}

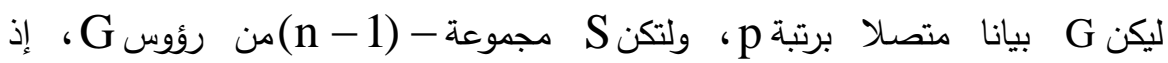

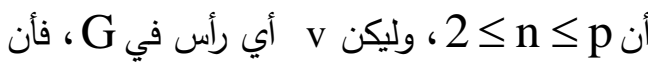

$\left.\mathrm{d}_{\mathrm{n}}(\mathrm{v}, \mathrm{S})=\min \{\mathrm{d}(\mathrm{v}, \mathrm{u}): \mathrm{u} \in \mathrm{S}\}\right)$

$$
\begin{aligned}
& \mathrm{d}_{\mathrm{n}}(\mathrm{v}, \mathrm{S})=0 \text {; } \\
& \text { عندما V viأن } \\
& \mathrm{d}_{\mathrm{n}}(\mathrm{v}, \mathrm{S}) \geq 1 \text {; } \\
& \text { عندما V v }
\end{aligned}
$$

$$
\text { التعريف(2.2) : }
$$

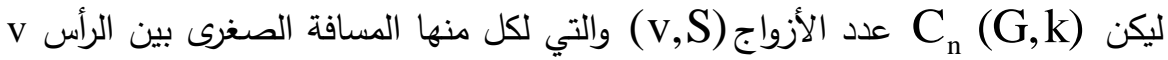

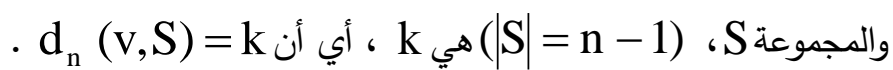

الآن نعرف متعددة حدود وينر n- للبيان بأنها متعددة الحدود

$$
\begin{aligned}
& \mathrm{W}_{\mathrm{n}}(\mathrm{G} ; \mathrm{x})=\sum_{\mathrm{k}=0}^{\delta_{\mathrm{n}}} \mathrm{C}_{\mathrm{n}}(\mathrm{G}, \mathrm{k}) \mathrm{x}^{\mathrm{k}}
\end{aligned}
$$

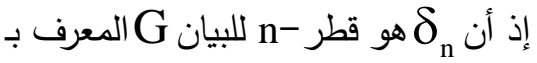

$$
\delta_{\mathrm{n}}=\operatorname{diam}_{\mathrm{n}}(\mathrm{G})=\max \{\mathrm{d}(\mathrm{v}, \mathrm{S}): \mathrm{v} \in \mathrm{V}, \mathrm{S} \subseteq \mathrm{V},|\mathrm{S}|=\mathrm{n}-1\}
$$

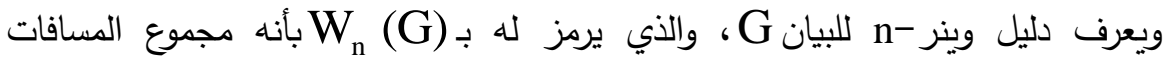

$$
\mathrm{W}_{\mathrm{n}}(\mathrm{G})=\sum_{\substack{(\mathrm{v}, \mathrm{S}) \\ \mathrm{v} \in \mathrm{V}, \mathrm{S} \subseteq \mathrm{V}}} \mathrm{d}_{\mathrm{n}}(\mathrm{v}, \mathrm{S})
$$$$
\text { الصغرى لكل الأزواج (v,S) في البيان G ف و أي أن }
$$

$$
\text { إذ أن } \mid \text { إن }
$$

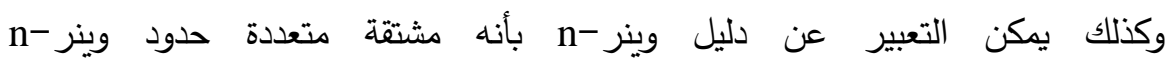

$$
\begin{aligned}
& \text { ، X = = عندما X X } \\
& \text { أي أن }
\end{aligned}
$$

$$
\mathrm{W}_{\mathrm{n}}(\mathrm{G})=\left.\mathrm{W}_{\mathrm{n}}^{\prime}(\mathrm{G} ; \mathrm{x})\right|_{\mathrm{x}=1}=\sum_{\mathrm{k}=1}^{\delta_{\mathrm{n}}} \mathrm{k} \mathrm{C}_{\mathrm{n}}(\mathrm{G}, \mathrm{k})
$$

ومن المفيد جدا ولأجل تسهيل كثير من المعالجات لمتعددات حدود وينر -n سوف نذكر

$$
\text { بعض خواص معاملات متعددات حدود وينر مئ }
$$




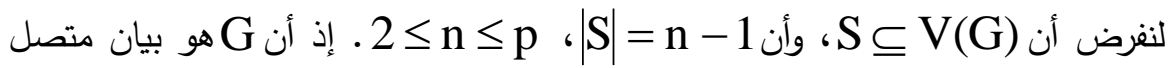

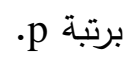

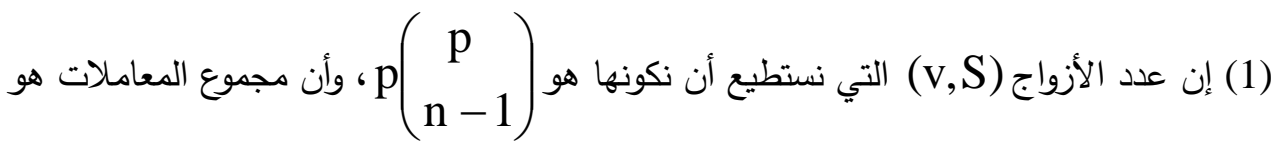
$\sum_{i=1}^{\delta_{n}} C_{n}(G, i)=(p-n+1)\left(\begin{array}{c}p \\ n-1\end{array}\right)=p\left(\begin{array}{l}p-1 \\ n-1\end{array}\right)$

$\mathrm{C}_{\mathrm{n}}(\mathrm{G}, 0)=\mathrm{p}\left(\begin{array}{l}\mathrm{p}-1 \\ \mathrm{n}-2\end{array}\right)$

هو W

\section{C (G,1) المأخوذة الآتية تعين لنا قيمة الععامل (3)}

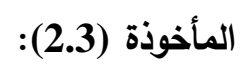

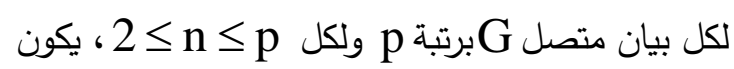

$$
\mathrm{C}_{\mathrm{n}}(\mathrm{G}, 1)=\mathrm{p}\left(\begin{array}{l}
\mathrm{p}-1 \\
\mathrm{n}-1
\end{array}\right)-\sum_{\forall v \in \mathrm{V}}\left(\begin{array}{c}
\mathrm{p}-1-\operatorname{deg}_{\mathrm{G}} \mathrm{v} \\
\mathrm{n}-1
\end{array}\right) \text {; }
$$

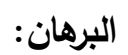

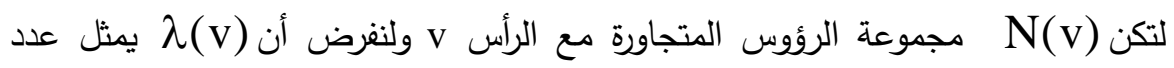

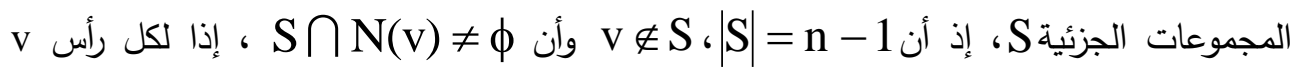

$$
\begin{aligned}
\lambda(\mathrm{v})=\left(\begin{array}{l}
\mathrm{p}-1 \\
\mathrm{n}-1
\end{array}\right)-\left(\begin{array}{c}
\mathrm{p}-1-\operatorname{deg}_{\mathrm{G}} \mathrm{v} \\
\mathrm{n}-1
\end{array}\right) \\
\mathrm{C}_{\mathrm{n}}(\mathrm{G}, 1)=\sum_{\mathrm{v} \in \mathrm{v}}\left\{\left(\begin{array}{l}
\mathrm{p}-1 \\
\mathrm{n}-1
\end{array}\right)-\left(\begin{array}{c}
\mathrm{p}-1-\operatorname{deg}_{\mathrm{G}} \mathrm{v} \\
\mathrm{n}-1
\end{array}\right)\right\} \\
=\mathrm{p}\left(\begin{array}{l}
\mathrm{p}-1 \\
\mathrm{n}-1
\end{array}\right)-\sum_{\mathrm{v} \in \mathrm{V}}\left(\begin{array}{c}
\mathrm{p}-1-\operatorname{deg}_{\mathrm{G}} \mathrm{v} \\
\mathrm{n}-1
\end{array}\right)
\end{aligned}
$$

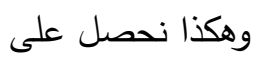

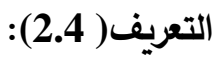




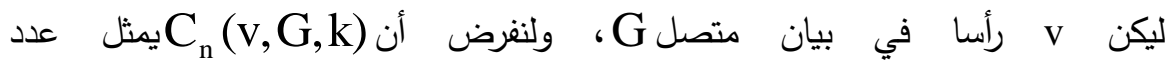

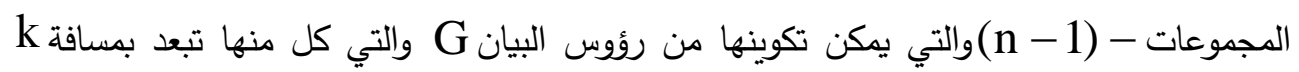

$$
\begin{aligned}
& \text { عن الرأسv. إذ أن k } \\
& \text { واضح من التعريف أعلاه أن } \\
& \sum_{\mathrm{v} \in \mathrm{V}} \mathrm{C}_{\mathrm{n}}(\mathrm{v}, \mathrm{G}, \mathrm{k})=\mathrm{C}_{\mathrm{n}}(\mathrm{G}, \mathrm{k}) \\
& \mathrm{k} \geq 0 \text { كل } \\
& \text { ونعرف متعددة حدود وينر -n بالنسبة إلى الرأس V بـ } \\
& \mathrm{W}_{\mathrm{n}}(\mathrm{v}, \mathrm{G} ; \mathrm{x})=\sum_{\mathrm{k}=0}^{\delta_{\mathrm{n}}} \mathrm{C}_{\mathrm{n}}(\mathrm{v}, \mathrm{G}, \mathrm{k}) \mathrm{x}^{\mathrm{k}}
\end{aligned}
$$

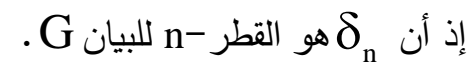

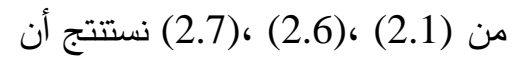

$\sum_{\mathrm{v} \in \mathrm{V}} \mathrm{W}_{\mathrm{n}}(\mathrm{v}, \mathrm{G} ; \mathrm{x})=\mathrm{W}_{\mathrm{n}}(\mathrm{G} ; \mathrm{x})$

والتي يمكن إثباتها بسهولة كالأتي:

بما أن

$$
\begin{aligned}
& \mathrm{W}_{\mathrm{n}}(\mathrm{v}, \mathrm{G} ; \mathrm{x})=\sum_{\mathrm{k}=0}^{\delta_{\mathrm{n}}} \mathrm{C}_{\mathrm{n}}(\mathrm{v}, \mathrm{G}, \mathrm{k}) \mathrm{x}^{\mathrm{k}} \\
& \text { وبأخذ المجموع للطرفين لكل رأس v في V(G) نحصل على V } \\
& \sum_{\mathrm{v} \in \mathrm{V}} \mathrm{W}_{\mathrm{n}}(\mathrm{v}, \mathrm{G} ; \mathrm{x})=\sum_{\mathrm{v} \in \mathrm{V}} \sum_{\mathrm{k}=0}^{\delta_{\mathrm{n}}} \mathrm{C}_{\mathrm{n}}(\mathrm{v}, \mathrm{G}, \mathrm{k}) \mathrm{x}^{\mathrm{k}} \\
& =\sum_{\mathrm{k}=0}^{\delta_{\mathrm{n}}} \sum_{\mathrm{v} \in \mathrm{V}} \mathrm{C}_{\mathrm{n}}(\mathrm{v}, \mathrm{G}, \mathrm{k}) \mathrm{x}^{\mathrm{k}} \\
& =\sum_{\mathrm{k}=0}^{\delta_{\mathrm{n}}} \mathrm{C}_{\mathrm{n}}(\mathrm{G}, \mathrm{k}) \mathrm{x}^{\mathrm{k}} \\
& =\mathrm{W}_{\mathrm{n}}(\mathrm{G} ; \mathrm{x})
\end{aligned}
$$

n - لبعض البيانات الخاصة:

\section{n-Wiener Polynomials of some Special Graphs:}

نلاحظ في إيجاد متعددات حدود وينر نسبة للمسافة الاعتيادية ، أن بالإمكان الحصول

على صيغة للحدودية عندما يكون للبيان انتظام معين بحيث يمكن حساب الصيغة العامة

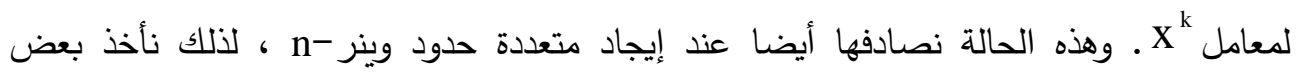


n- البيانات الخاصة لأن لها انتظاماً كبيراً في تراكيبها ـ وقد أوجدنا في هذا البند متعدة حدود وينان

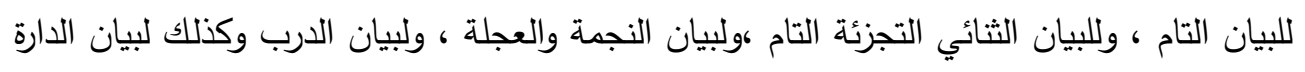

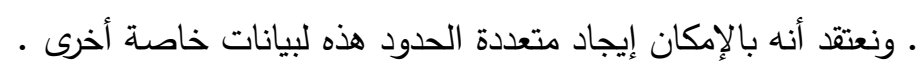

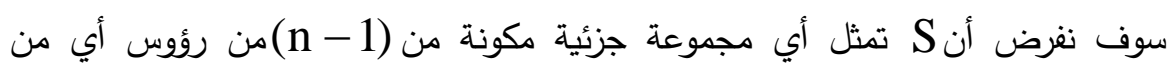

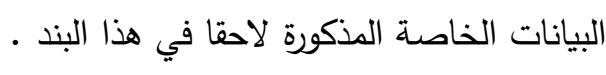

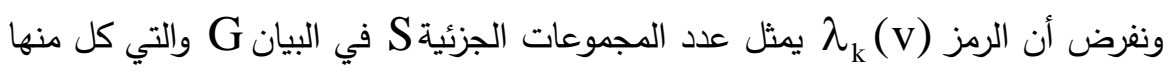

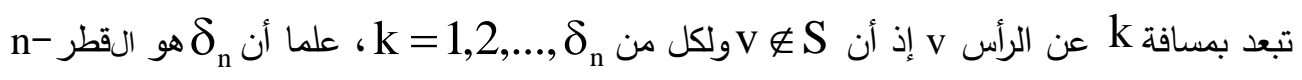

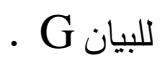
$\mathrm{C}_{\mathrm{n}}(\mathrm{G}, \mathrm{k})=\sum_{\mathrm{v} \in \mathrm{V}} \lambda_{\mathrm{k}}(\mathrm{v})$

من السهولة ملاحظة أن

\section{Complete and Complete bipartite graphs:}

بيان تام وبيان ثنائي التجزئة تام:

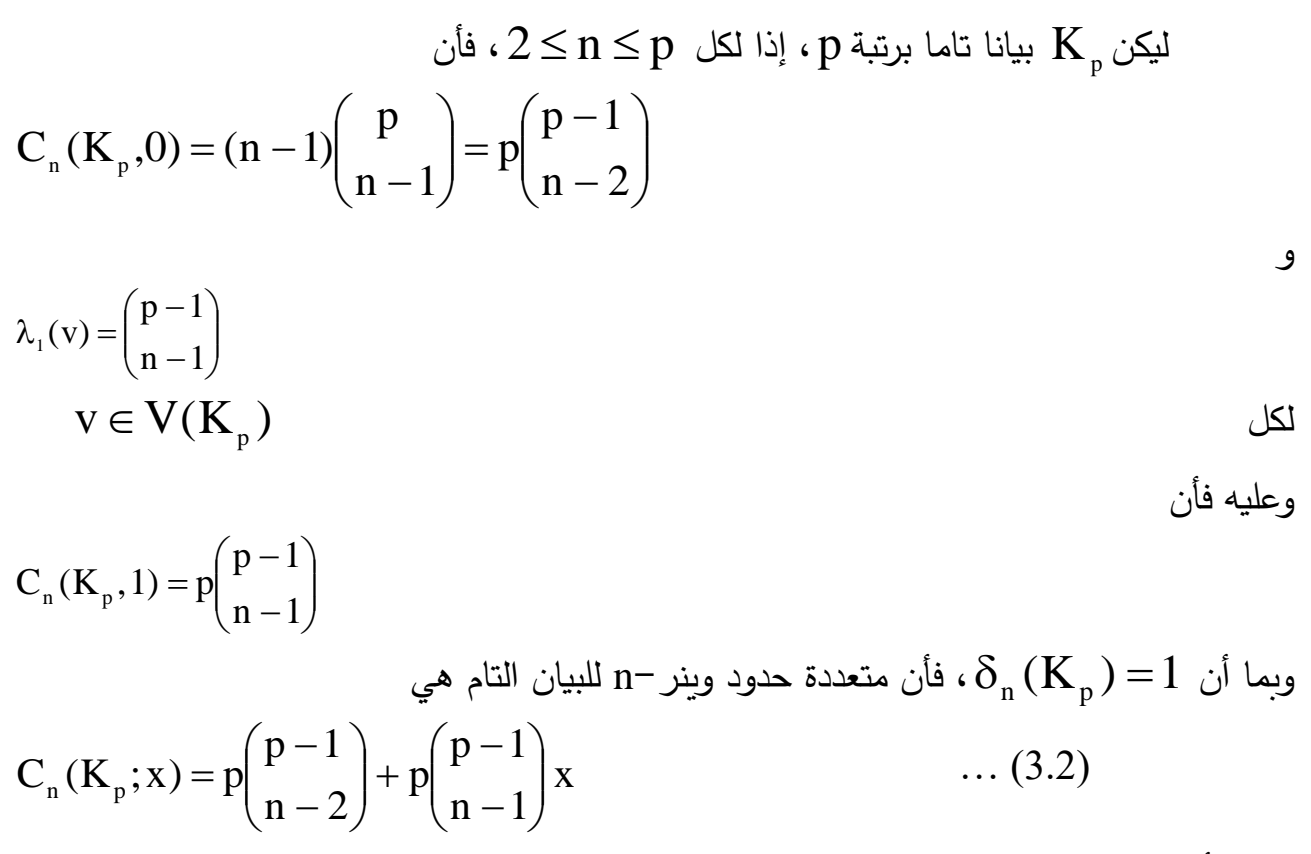

لاحظ أن

$\mathrm{W}_{2}\left(\mathrm{~K}_{\mathrm{p}} ; \mathrm{x}\right)=\mathrm{p}+\mathrm{p}(\mathrm{p}-1) \mathrm{x} \neq \mathrm{W}\left(\mathrm{K}_{\mathrm{P}} ; \mathrm{x}\right)=\mathrm{p}+\frac{1}{2} \mathrm{p}(\mathrm{p}-1) \mathrm{x}$ 
وذلك بسبب كون الزوج المرتب (v, $)$ لا يتطابق مع الزوج (u,\{v\}) عندما

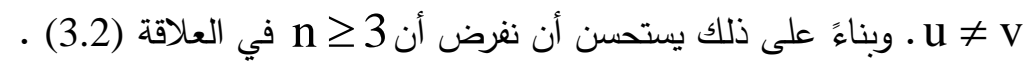

واضح أن دليل وينر n- للبيان التام

$\mathrm{W}_{\mathrm{n}}\left(\mathrm{K}_{\mathrm{p}}\right)=\mathrm{p}\left(\begin{array}{l}\mathrm{p}-1 \\ \mathrm{n}-1\end{array}\right)$

الآن نأخذ البيان الثنائي التجزئة التام K K $_{\alpha, \beta}$ إذ أن مجموعتي التجزئة للرؤوس هما :

$\mathrm{V}_{1}=\left\{\mathrm{v}_{1}, \mathrm{v}_{2}, \ldots, \mathrm{v}_{\alpha}\right\}$,

$\mathrm{V}_{2}=\left\{\mathrm{v}_{\alpha+1}, \mathrm{v}_{\alpha+2}, \ldots, \mathrm{v}_{\alpha+\beta}\right\}$.

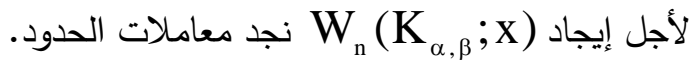
من العلاقة (2.5) فأن

$\mathrm{C}_{\mathrm{n}}\left(\mathrm{K}_{\alpha, \beta}, 0\right)=\mathrm{p}\left(\begin{array}{l}\mathrm{p}-1 \\ \mathrm{n}-2\end{array}\right) ; \quad \mathrm{p}=\alpha+\beta$

ومن المأخوذة (2.3) ، نحصل على

$\mathrm{C}_{\mathrm{n}}\left(\mathrm{K}_{\alpha, \beta}, 1\right)=\mathrm{p}\left(\begin{array}{c}\mathrm{p}-1 \\ \mathrm{n}-1\end{array}\right)-\sum_{\mathrm{v} \in \mathrm{V}\left(\mathrm{K}_{\alpha, \beta}\right)}\left(\begin{array}{c}\mathrm{p}-1-\operatorname{deg} \mathrm{V} \\ \mathrm{n}-1\end{array}\right)$

$$
=p\left(\begin{array}{l}
p-1 \\
n-1
\end{array}\right)-\left\{\alpha\left(\begin{array}{l}
\alpha-1 \\
n-1
\end{array}\right)+\beta\left(\begin{array}{l}
\beta-1 \\
n-1
\end{array}\right)\right\}
$$

$\mathrm{C}_{\mathrm{n}}\left(\mathrm{K}_{\alpha, \beta}, 1\right)+\mathrm{C}_{\mathrm{n}}\left(\mathrm{K}_{\alpha, \beta}, 2\right)=\mathrm{p}\left(\begin{array}{l}\mathrm{p}-1 \\ \mathrm{n}-1\end{array}\right)$

وبما أن

لأن قطر n- للبيان الثنائي التجزئة التام هو 2 ، فأن

$\mathrm{C}_{\mathrm{n}}\left(\mathrm{K}_{\alpha, \beta}, 2\right)=\alpha\left(\left(\begin{array}{l}\alpha-1 \\ \mathrm{n}-1\end{array}\right)+\beta\left(\begin{array}{l}\beta-1 \\ \mathrm{n}-1\end{array}\right)\right.$

وبهذا نحصل مما تقدم على العبارة الآتية:

(3.1) : (3.1) :

لكل

التجزئة التام 


$$
\begin{aligned}
\mathrm{W}_{\mathrm{n}}\left(\mathrm{K}_{\alpha, \beta} ; \mathrm{x}\right) & =\mathrm{p}\left(\begin{array}{l}
\mathrm{p}-1 \\
\mathrm{n}-2
\end{array}\right)+\left\{\mathrm{p}\left(\begin{array}{l}
\mathrm{p}-1 \\
\mathrm{n}-1
\end{array}\right)-\alpha\left(\begin{array}{l}
\alpha-1 \\
\mathrm{n}-1
\end{array}\right)-\beta\left(\begin{array}{l}
\beta-1 \\
\mathrm{n}-1
\end{array}\right)\right\} \mathrm{x} \\
& +\left\{\alpha\left(\begin{array}{l}
\alpha-1 \\
\mathrm{n}-1
\end{array}\right)+\beta\left(\begin{array}{l}
\beta-1 \\
\mathrm{n}-1
\end{array}\right)\right\} \mathrm{x}^{2}
\end{aligned}
$$

$$
\text { p }=\alpha+\beta \text { إذ أن }
$$

واضح أن دليل وينر -n للبيان

$\mathrm{W}_{\mathrm{n}}\left(\mathrm{K}_{\alpha, \beta}\right)=\mathrm{p}\left(\begin{array}{l}\mathrm{p}-1 \\ \mathrm{n}-1\end{array}\right)+\alpha\left(\begin{array}{l}\alpha-1 \\ \mathrm{n}-1\end{array}\right)+\beta\left(\begin{array}{l}\beta-1 \\ \mathrm{n}-1\end{array}\right)$

\section{Star and Wheel graphs:}

(3.2) (بيان نجمة وبيان عجلة:

(3.2) - العبارة (3):

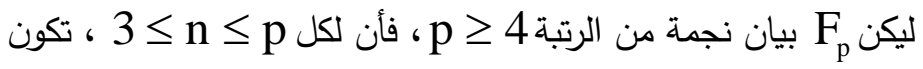

$\mathrm{W}_{\mathrm{n}}\left(\mathrm{F}_{\mathrm{p}} ; \mathrm{x}\right)=\mathrm{p}\left(\begin{array}{l}\mathrm{p}-1 \\ \mathrm{n}-2\end{array}\right)+\mathrm{n}\left(\begin{array}{l}\mathrm{p}-1 \\ \mathrm{n}-1\end{array}\right) \mathrm{x}+(\mathrm{p}-1)\left(\begin{array}{l}\mathrm{p}-2 \\ \mathrm{n}-1\end{array}\right) \mathrm{x}^{2}$

$\mathrm{W}_{\mathrm{n}}\left(\mathrm{F}_{\mathrm{p}}\right)=(2 \mathrm{p}-\mathrm{n})\left(\begin{array}{l}\mathrm{p}-1 \\ \mathrm{n}-1\end{array}\right)$

بما أن النجمة Fي بيان ثنائي التجزئة

العلاقتين (3.4 ) و(3.5 ) ،وإجراء بعض العمليات التبسيطية نحصل على العلاقتين (3.6 ) و (3.7) على الترتيب .

والآن نأخذ بيان العجلة E بالرتبة p 4 p المبينة في الثكل(1-3).

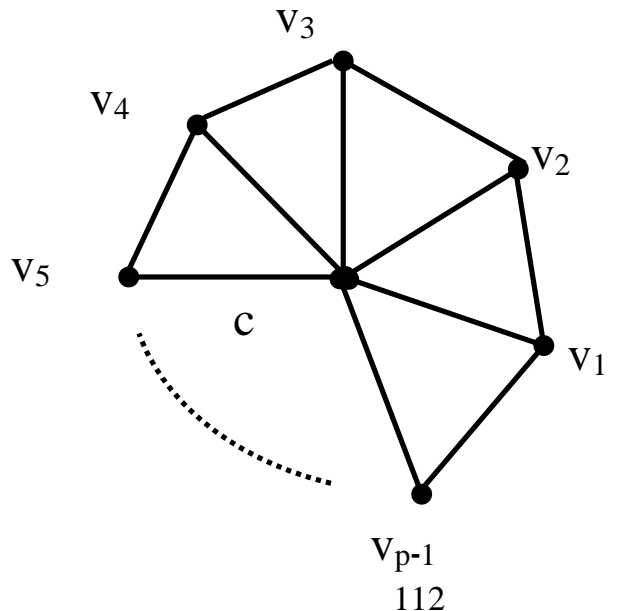




$$
\begin{aligned}
& \text { الثكل (1-3) بيان العجلة }
\end{aligned}
$$

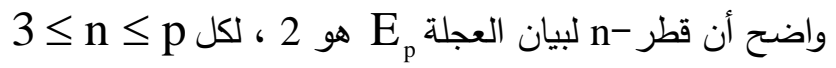

$$
\begin{aligned}
& \text { وعليه فأن } \\
& \mathrm{C}_{\mathrm{n}}\left(\mathrm{E}_{\mathrm{p}}, 0\right)=\mathrm{p}\left(\begin{array}{l}
\mathrm{p}-1 \\
\mathrm{n}-2
\end{array}\right) \\
& \mathrm{C}_{\mathrm{n}}\left(\mathrm{E}_{\mathrm{p}}, 1\right)=\mathrm{p}\left(\begin{array}{c}
\mathrm{p}-1 \\
\mathrm{n}-1
\end{array}\right)-(\mathrm{p}-1)\left(\begin{array}{c}
\mathrm{p}-4 \\
\mathrm{n}-1
\end{array}\right) \text {; } \\
& C_{n}\left(E_{p}, 2\right)=(p-1)\left(\begin{array}{l}
p-4 \\
n-1
\end{array}\right) \text {. }
\end{aligned}
$$

وهكذا نحصل على العبارة الآتية .

$$
\text { العبارة (3.3): }
$$

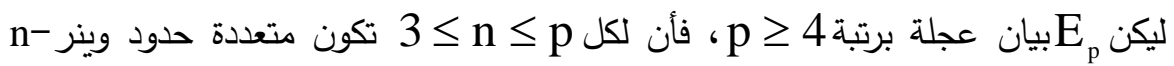

$$
\text { E لـ الصيغة الآتية: }
$$

$$
\begin{aligned}
& W_{n}\left(E_{p} ; x\right)=p\left(\begin{array}{l}
p-1 \\
n-2
\end{array}\right)+\left\{p\left(\begin{array}{l}
p-1 \\
n-1
\end{array}\right)-(p-1)\left(\begin{array}{l}
p-4 \\
n-1
\end{array}\right)\right\} \mathrm{x}+(\mathrm{p}-1)\left(\begin{array}{l}
\mathrm{p}-4 \\
\mathrm{n}-1
\end{array}\right) \mathrm{x}^{2} \\
& \mathrm{~W}_{\mathrm{n}}\left(\mathrm{E}_{\mathrm{p}}\right)=\mathrm{p}\left(\begin{array}{l}
\mathrm{p}-1 \\
\mathrm{n}-1
\end{array}\right)+(\mathrm{p}-1)\left(\begin{array}{l}
\mathrm{p}-4 \\
\mathrm{n}-1
\end{array}\right)
\end{aligned}
$$

Path graph:

ليكن R بيان درب برتبة p كما هو مبين في الثكل(2-3).
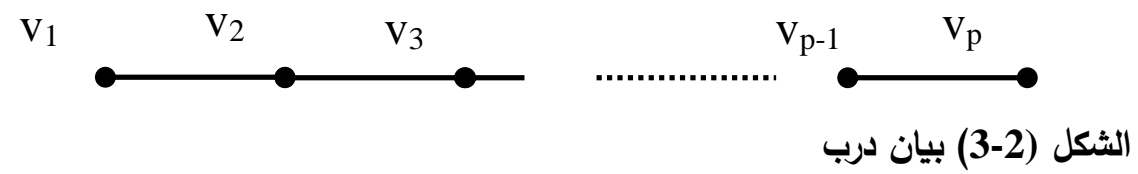

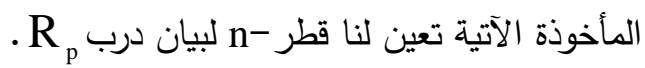

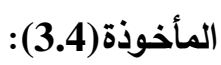

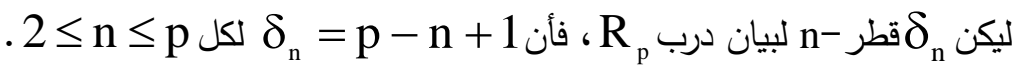


واضح أنه عندما

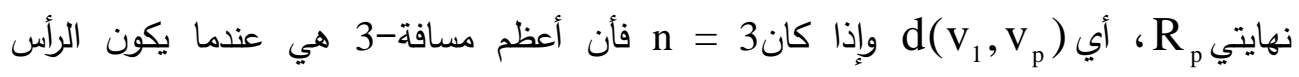

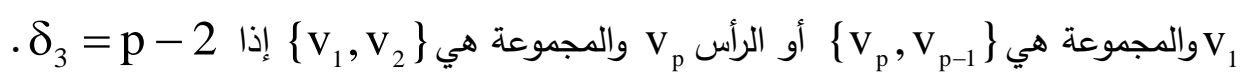

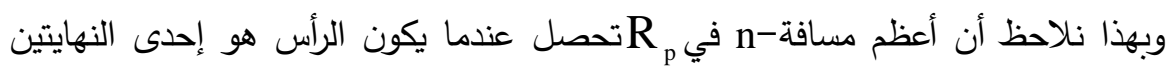

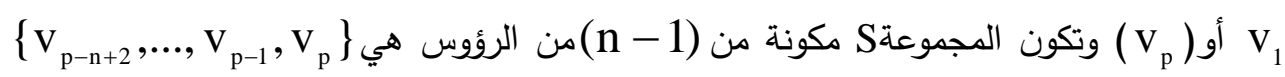
. ( $\left.\left\{\mathrm{v}_{1}, \mathrm{v}_{2}, \ldots, \mathrm{v}_{\mathrm{n}-1}\right\}\right)$ وعليه فأن

$\delta_{\mathrm{n}}=\max _{(\mathrm{v}, \mathrm{S})} \mathrm{d}_{\mathrm{n}}(\mathrm{v}, \mathrm{S})=\mathrm{p}-(\mathrm{n}-1)$

$$
\text { وبهذا يتم البرهان }
$$

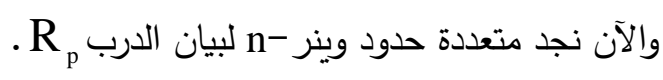

المبرهنة(3.5) :

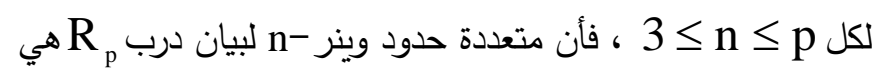

$\mathrm{W}_{\mathrm{n}}\left(\mathrm{R}_{\mathrm{p}} ; \mathrm{x}\right)=\mathrm{p}\left(\begin{array}{l}\mathrm{p}-1 \\ \mathrm{n}-2\end{array}\right)+\sum_{\mathrm{k}=1}^{\mathrm{p}-\mathrm{n}+1} \mathrm{C}_{\mathrm{n}}\left(\mathrm{R}_{\mathrm{p}}, \mathrm{k}\right) \mathrm{x}^{\mathrm{k}}$

إذ أن لكل1)

$\mathrm{C}_{\mathrm{n}}\left(\mathrm{R}_{\mathrm{p}}, \mathrm{k}\right)=\left(\begin{array}{c}\mathrm{p}-2 \mathrm{k} \\ 1\end{array}\right)\left\{2\left(\begin{array}{c}\mathrm{p}-2 \mathrm{k}-1 \\ \mathrm{n}-2\end{array}\right)+\left(\begin{array}{c}\mathrm{p}-2 \mathrm{k}-1 \\ \mathrm{n}-3\end{array}\right)\right\}+2 \sum_{\mathrm{i}=\mathrm{k}+1}^{2 \mathrm{k}}\left(\begin{array}{l}\mathrm{p}-\mathrm{i} \\ \mathrm{n}-2\end{array}\right)$

من المأخوذة (3.4) ، فأن قطر - للبيان Rو

$\mathrm{C}_{\mathrm{n}}\left(\mathrm{R}_{\mathrm{p}}, 0\right)=\mathrm{p}\left(\begin{array}{l}\mathrm{p}-1 \\ \mathrm{n}-2\end{array}\right)$

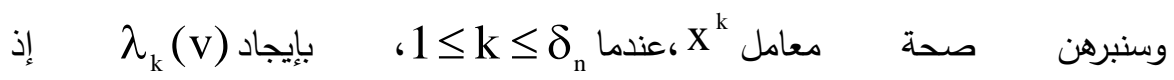

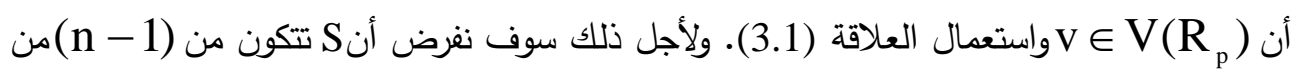
الرؤس ولا يحتوي على الرأس v v 


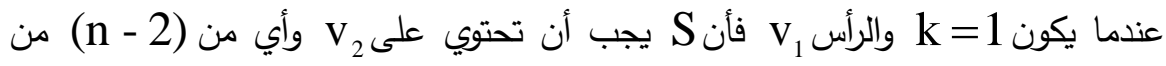

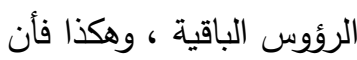
$\lambda_{1}\left(\mathrm{v}_{1}\right)=\left(\begin{array}{l}\mathrm{p}-2 \\ \mathrm{n}-2\end{array}\right)$

$$
\lambda_{1}\left(\mathrm{v}_{\mathrm{p}}\right)=\left(\begin{array}{l}
\mathrm{p}-2 \\
\mathrm{n}-2
\end{array}\right)
$$

وبالمثل فأن

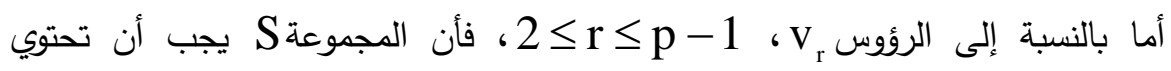
$\lambda_{1}\left(\mathrm{v}_{\mathrm{r}}\right)=2\left(\begin{array}{l}\mathrm{p}-3 \\ \mathrm{n}-2\end{array}\right)+\left(\begin{array}{l}\mathrm{p}-3 \\ \mathrm{n}-3\end{array}\right), 2 \leq \mathrm{r} \leq \mathrm{p}$

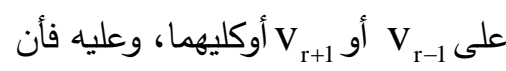
$\mathrm{C}_{\mathrm{n}}\left(\mathrm{R}_{\mathrm{p}}, 1\right)=2\left(\begin{array}{l}\mathrm{p}-2 \\ \mathrm{n}-2\end{array}\right)+\left(\begin{array}{c}\mathrm{p}-2 \\ 1\end{array}\right)\left\{2\left(\begin{array}{l}\mathrm{p}-3 \\ \mathrm{n}-2\end{array}\right)+\left(\begin{array}{l}\mathrm{p}-3 \\ \mathrm{n}-3\end{array}\right)\right\}$

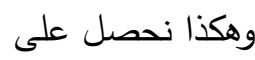

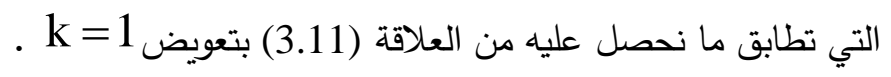

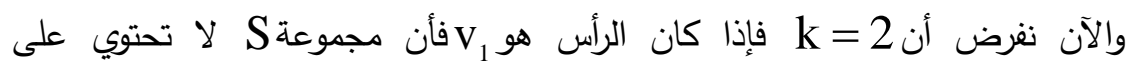

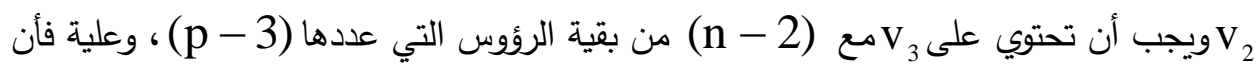
$\lambda_{2}\left(\mathrm{v}_{1}\right)=\left(\begin{array}{l}\mathrm{p}-3 \\ \mathrm{n}-2\end{array}\right)$

$\lambda_{2}\left(\mathrm{v}_{\mathrm{p}}\right)=\left(\begin{array}{l}\mathrm{p}-3 \\ \mathrm{n}-2\end{array}\right)$ وبالمثل بالنسبة إلى الرأس V ، أي أن وبالنسبة إلى الرأس V فأن S لا تحتوي على V و V V ويجب أن تحتوي على V ، وعليه $\lambda_{2}\left(\mathrm{v}_{2}\right)=\left(\begin{array}{l}\mathrm{p}-4 \\ \mathrm{n}-2\end{array}\right)$ $\lambda_{2}\left(\mathrm{v}_{\mathrm{p}-1}\right)=\left(\begin{array}{l}\mathrm{p}-4 \\ \mathrm{n}-2\end{array}\right)$ وبالمثل بالنسبة إلى الرأس V-1 v أي أن 


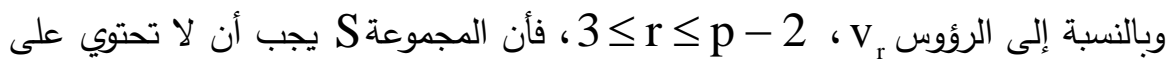

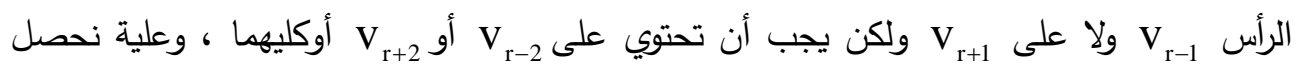
$\lambda_{2}\left(\mathrm{v}_{\mathrm{r}}\right)=2\left(\begin{array}{l}\mathrm{p}-5 \\ \mathrm{n}-2\end{array}\right)+\left(\begin{array}{l}\mathrm{p}-5 \\ \mathrm{n}-3\end{array}\right)$

$\mathrm{C}_{\mathrm{n}}\left(\mathrm{R}_{\mathrm{p}}, 2\right)=2\left(\begin{array}{l}\mathrm{p}-3 \\ \mathrm{n}-2\end{array}\right)+2\left(\begin{array}{l}\mathrm{p}-4 \\ \mathrm{n}-2\end{array}\right)+\left(\begin{array}{c}\mathrm{p}-4 \\ 1\end{array}\right)\left\{2\left(\begin{array}{l}\mathrm{p}-5 \\ \mathrm{n}-2\end{array}\right)+\left(\begin{array}{l}\mathrm{p}-5 \\ \mathrm{n}-3\end{array}\right)\right\}$

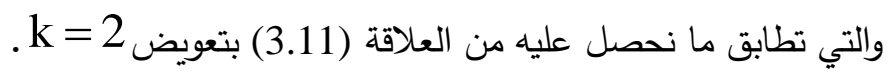

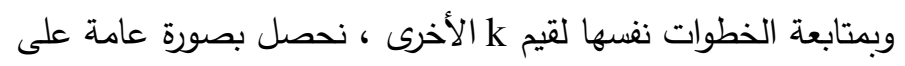
$\lambda_{\mathrm{k}}\left(\mathrm{v}_{\mathrm{i}}\right)=\lambda_{\mathrm{k}}\left(\mathrm{v}_{\mathrm{p}-\mathrm{i}+1}\right)=\left(\begin{array}{c}\mathrm{p}-\mathrm{k}-\mathrm{i} \\ \mathrm{n}-2\end{array}\right) ; \quad 1 \leq \mathrm{i} \leq \mathrm{k}$ $\lambda_{\mathrm{k}}\left(\mathrm{v}_{\mathrm{i}}\right)=2\left(\begin{array}{c}\mathrm{p}-2 \mathrm{k}-1 \\ \mathrm{n}-2\end{array}\right)+\left(\begin{array}{c}\mathrm{p}-2 \mathrm{k}-1 \\ \mathrm{n}-3\end{array}\right) ; \mathrm{k}+1 \leq \mathrm{i} \leq \mathrm{p}-\mathrm{k}$

$\mathrm{C}_{\mathrm{n}}\left(\mathrm{R}_{\mathrm{p}}, \mathrm{k}\right)=\left(\begin{array}{c}\mathrm{p}-2 \mathrm{k} \\ 1\end{array}\right)\left\{2\left(\begin{array}{c}\mathrm{p}-2 \mathrm{k}-1 \\ \mathrm{n}-2\end{array}\right)+\left(\begin{array}{c}\mathrm{p}-2 \mathrm{k}-1 \\ \mathrm{n}-3\end{array}\right)\right\}+2 \sum_{\mathrm{i}=\mathrm{k}+1}^{2 \mathrm{k}}\left(\begin{array}{c}\mathrm{p}-\mathrm{i} \\ \mathrm{n}-2\end{array}\right)$

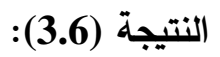

دليل وينر - لبيان درب Rو $\mathrm{W}_{\mathrm{n}}\left(\mathrm{R}_{\mathrm{p}}\right)=\sum_{\mathrm{k}=1}^{\mathrm{p}-\mathrm{n}+1} \mathrm{k}\left[\left(\begin{array}{c}\mathrm{p}-2 \mathrm{k} \\ 1\end{array}\right)\left\{\left(\begin{array}{c}\mathrm{p}-2 \mathrm{k}-1 \\ \mathrm{n}-2\end{array}\right)+\left(\begin{array}{c}\mathrm{p}-2 \mathrm{k}-1 \\ \mathrm{n}-3\end{array}\right)\right\}+2 \sum_{\mathrm{i}=\mathrm{k}+1}^{2 \mathrm{k}}\left(\begin{array}{c}\mathrm{p}-\mathrm{i} \\ \mathrm{n}-2\end{array}\right)\right]$

مباشرة يمكنا الحصول على العلاقة (3.12) وذلك باشتقاق الصيغة (3.10) بالنسبة

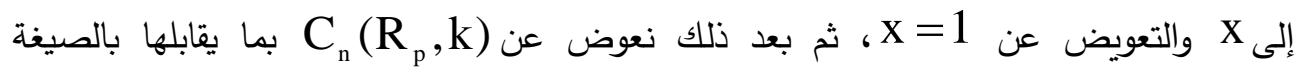




\section{Cycle graph:}

(3.4) بيان دارة:

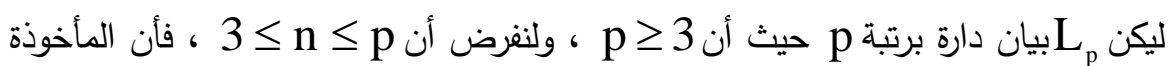

الآتية تعطينا قطر -n للبيان L المبين في الثكل(3-3).

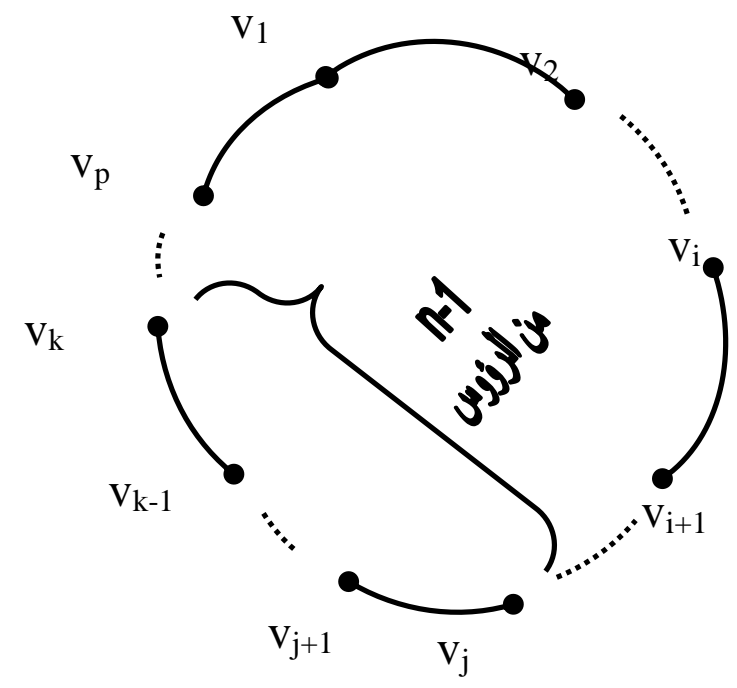

(3-3-3)

المأخوذة (3.7):

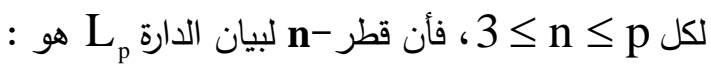

$\delta_{\mathrm{n}}=\delta_{\mathrm{n}}\left(\mathrm{L}_{\mathrm{p}}\right)=\left\lfloor\frac{\mathrm{p}-\mathrm{n}}{2}\right\rfloor+1$

البرهان:

لتكن

$$
\text { - d } \mathrm{d}_{\mathrm{n}}\left(\mathrm{v}_{\mathrm{i}}, \mathrm{S}\right)=\delta_{\mathrm{n}}\left(\mathrm{L}_{\mathrm{p}}\right)
$$

ولما كان القطر -n هو أعظم مسافة-n من رأس إلى مجموعة (n-1) من الرؤوس،

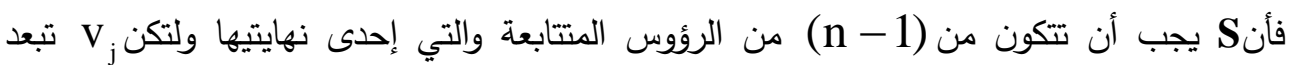

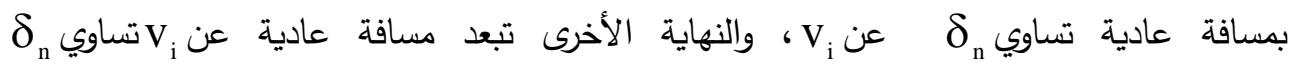

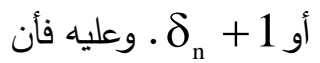




$$
\begin{aligned}
& \text { علي عزيز علي و أحمد محد علي } \\
& p=\left\{\begin{array}{l}
2\left(\delta_{n}-1\right)+(n-1)+1 \\
\left(2 \delta_{n}-1\right)+(n-1)+1
\end{array}\right. \\
& \mathrm{p}=\left\{\begin{array}{l}
2 \delta_{\mathrm{n}}-2+\mathrm{n} \\
2 \delta_{\mathrm{n}}-1+\mathrm{n}
\end{array}\right. \\
& \delta_{\mathrm{n}}=\left\{\begin{array}{l}
1+\frac{\mathrm{p}-\mathrm{n}}{2} \\
1+\frac{\mathrm{p}-\mathrm{n}-1}{2}
\end{array}\right. \\
& \delta_{\mathrm{n}}=\left\lfloor\frac{\mathrm{p}-\mathrm{n}}{2}\right\rfloor+1 \\
& \text { لاحظ النتيجة السابقة تتطابق مع نظيرتها في المسافة العادية ، أي عندما n =2. } \\
& \text { أو } \\
& \text { أي } \\
& \text { أو } \\
& \text { وبذلك فأن } \\
& \text { ولما كان }
\end{aligned}
$$

\section{المبرهنة (3.8) :}

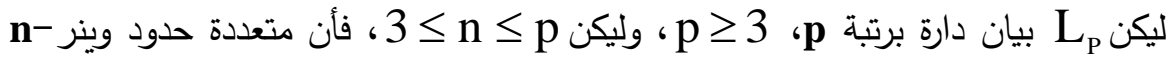

$$
\begin{aligned}
\mathrm{W}_{\mathrm{n}}\left(\mathrm{L}_{\mathrm{p}} ; \mathrm{x}\right) & =\mathrm{p}\left(\begin{array}{c}
\mathrm{p}-1 \\
\mathrm{n}-2
\end{array}\right)+\mathrm{p} \sum_{\mathrm{k}=1}^{\delta_{\mathrm{n}}}\left\{2\left(\begin{array}{c}
\mathrm{p}-2 \mathrm{k}-1 \\
\mathrm{n}-2
\end{array}\right)+\left(\begin{array}{c}
\mathrm{p}-2 \mathrm{k}-1 \\
\mathrm{n}-3
\end{array}\right)\right\} \mathrm{x}^{\mathrm{k}} \\
\delta_{\mathrm{n}}=\left\lfloor\frac{\mathrm{p}-\mathrm{n}}{2}\right\rfloor+1 & \text { البرهان: }
\end{aligned}
$$


$\mathrm{C}_{\mathrm{n}}\left(\mathrm{L}_{\mathrm{p}}, 0\right)=\mathrm{p}\left(\begin{array}{l}\mathrm{p}-1 \\ \mathrm{n}-2\end{array}\right)$

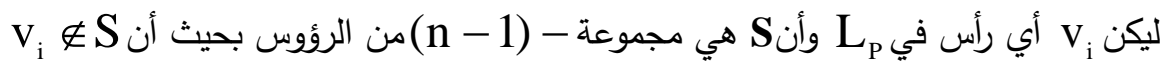

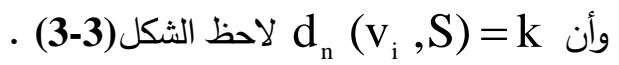

إذا S لا تحتوي على أي من الرؤوس في المجموعة

$\mathrm{U}=\left\{\mathrm{v}_{\mathrm{i}}, \mathrm{v}_{\mathrm{i}-1}, \ldots, \mathrm{v}_{\mathrm{i}-\mathrm{k}+1}, \mathrm{v}_{\mathrm{i}+1}, \mathrm{v}_{\mathrm{i}+2}, \ldots, \mathrm{v}_{\mathrm{i}+\mathrm{k}-1}\right\}$

ولكن S يجب أن تحتوي إما على V أو على V أو تحتوي على كليهما. وبذلك فأن

هنالك ثلاث حالات للمجموعة S وهي :

$\mathrm{v}_{\mathrm{i}+\mathrm{k}} \in \mathrm{S}, \mathrm{v}_{\mathrm{i}-\mathrm{k}} \notin \mathrm{S}$;

$\mathrm{S} \subseteq \mathrm{V}\left(\mathrm{L}_{\mathrm{P}}\right)-\left[\mathrm{U} \cup\left\{\mathrm{v}_{\mathrm{i}-\mathrm{k}}\right\}\right]$

$\mathrm{v}_{\mathrm{i}-\mathrm{k}} \in \mathrm{S}, \mathrm{v}_{\mathrm{i}+\mathrm{k}} \notin \mathrm{S}$;

$\mathrm{S} \subseteq \mathrm{V}\left(\mathrm{L}_{\mathrm{P}}\right)-\left[\mathrm{U} \cup\left\{\mathrm{V}_{\mathrm{i}+\mathrm{k}}\right\}\right]$

$\mathrm{v}_{\mathrm{i}-\mathrm{k}}, \mathrm{v}_{\mathrm{i}+\mathrm{k}} \in \mathrm{S}$;

$\mathrm{S} \subseteq \mathrm{V}\left(\mathrm{L}_{\mathrm{P}}\right)-\mathrm{U}$

$$
\text { وبما أن }|\mathrm{U}|=2 \mathrm{k}-1
$$

واستنادا إلى هذه الحالات فأن عدد هكذا مجموعات هو:

$\lambda_{\mathrm{k}}\left(\mathrm{v}_{\mathrm{i}}\right)=2\left(\begin{array}{c}\mathrm{p}-(2 \mathrm{k}+1) \\ \mathrm{n}-2\end{array}\right)+\left(\begin{array}{c}\mathrm{p}-(2 \mathrm{k}+1) \\ \mathrm{n}-3\end{array}\right), 1 \leq \mathrm{i} \leq \mathrm{p}$

واستنادا إلى العلاقة (3.1)، فأن لكل 1 1 1

$\mathrm{C}_{\mathrm{n}}\left(\mathrm{L}_{\mathrm{p}}, \mathrm{k}\right)=\mathrm{p}\left\{2\left(\begin{array}{c}\mathrm{p}-2 \mathrm{k}-1 \\ \mathrm{n}-2\end{array}\right)+\left(\begin{array}{c}\mathrm{p}-2 \mathrm{k}-1 \\ \mathrm{n}-3\end{array}\right)\right\}$

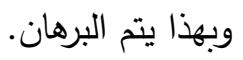

النتيجة (3.9):

دليل وينر - لبيان دارة p برتبة pe معطى بالصيغة الأتية:

$\mathrm{W}_{\mathrm{n}}\left(\mathrm{L}_{\mathrm{p}}\right)=\mathrm{p} \sum_{\mathrm{k}=1}^{\delta_{\mathrm{n}}} \mathrm{k}\left\{2\left(\begin{array}{c}\mathrm{p}-2 \mathrm{k}-1 \\ \mathrm{n}-2\end{array}\right)+\left(\begin{array}{c}\mathrm{p}-2 \mathrm{k}-1 \\ \mathrm{n}-3\end{array}\right)\right\}$

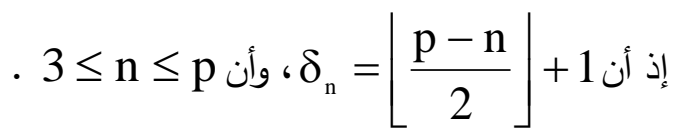

البرهان:

باشتقاق متعددة حدود وينر -n لبيان الدارة L بالنسبة إلى X

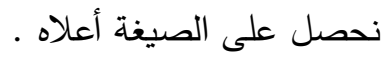


ملي اجستير .جامعة الموصل عمد علي (2005) متعددات حدود وينر لتعميم المسافة في البيانات رسالة

[3] Buckley F. and F. Harary; (1990) Distance in Graphs. Addison Wesley, New York.

[4] Dankelmann, P.; W. Goddard; M. A. Henning and H. C. Swart, (1999) "Generalized eccentricity, radius and diameter in graphs", Foundation for Research Development. c John Wiely \& Sons, Inc. Networks 34: 312-319. 